\title{
Neumonía en el anciano: ¿es atípica su presentación clínica?
}

\author{
S. GONZÁLEZ QUIJADA, M. GALDÓS BARROSO, A. RIEGO VALLEDOR, \\ G. FERNÁNDEZ ÁLVAREZ*, $M^{\mathrm{a}}$. C. GALINDO VICENTE** \\ Sección de Medicina Interna. *Servicio de Radiodiagnóstico. Hospital General Yagüe. \\ Burgos.
}

\author{
PNEUMONIA IN THE AGED PATIENT: IS THE CLINICAL \\ PRESENTATION ATYPICAL?
}

\section{RESUMEN}

Objetivo: Recientemente, varios estudios han puesto en duda el carácter frecuentemente atípico de la clínica en la neumonía del anciano. Por ello, estudiamos las formas de presentación clínica de esta patología en pacientes de mayor edad.

Material y métodos: Revisión retrospectiva de 179 casos de neumonía en pacientes mayores de 65 años, ingresados en nuestro hospital en el año 1992.

Resultados: Los pacientes presentaban una edad media de 78 años, siendo la proporción de varones del 65,9\%. El 91\% presentaban neumonías adquiridas en la comunidad. El $73,1 \%$ mostraron un presentación clínica dominante típica (febril-respiratoria); respiratoria $(47,4 \%)$, febril $(25,7 \%)$. El resto presentaron patrones atípicos; mental-neurológico o de "disfunción cerebral" $(10,6 \%)$, deambulación-estado general $(6,15 \%)$ y abdominal-digestivo (9,5\%). El patrón respiratorio se asoció con la presencia de antecedentes respiratorios $(\mathrm{p}<0,001)$, el febril con la ausencia de antecedentes cardiovasculares $(\mathrm{p}<0,05)$, el mental-neurológico con la presencia de antecedentes neurológicos $(\mathrm{p}<0,001)$ y la alteración de la deambulación-estado general con un tiempo de evolución mayor de 7 días $(\mathrm{p}<0,05)$.

Conclusión: La mayoría de nuestros pacientes mayores de 65 años con neumonía cursan con un patrón clínico típico (febril-respiratorio). La patología previa del paciente condiciona la forma de presentación clínica.

PALABRAS CLAVE: Neumonía. Ancianos. Clínica.

\begin{abstract}
Objective: Some new studies have questioned the often atypical cha racter of pneumonia in aged patients. Therefore to study the different cli nical presentation of this patology is our aim.

Material and methods: Retrospective analysis of 179 cases of pneu monia in patients aged older than 65 years who were admitted in our hospital during 1992.

Results: The average age of the patients was 78 years, being $65.9 \%$ of them men. $91 \%$ presented pneumonias adquired in the community. $73.1 \%$ showed a typical dominant clinical presentation (febrile-respira tory); respiratory (47.4\%), febrile (25.7\%). The rest presented atypical patterns; mental-neurologic or "cerebral dysfunction" (10.6\%), peram bulation-general state $(6.15 \%)$ and digestive-abdominal (9.5\%). The res piratory pattern was associated to the presence of respiratory antece dents $(p<0.001)$; the febrile pattem to the absence of cardiovascular antecedents $(p<0.05)$; the mental-neurologic to the presence of neurolo gic antecedents $(p<0.001)$ and the alteration of perambulation-general state to an evolution of more than 7 days ( $p<0.05$ ).

Conclusions: The greatest number of our pneumonia patients older than 65 years follows a typical clinical pattern febrile-respiratory). The patient's prior pathology conditions the clinical presentation.
\end{abstract}

KEY WORDS: Pneumonia. Aged patients. Clinic.

González Quijada S, Galdós Barroso M, Riego Valledor A, Fernández. Alvarez G, Galindo Vicente Ma C. Neumonía en el anciano: ¿es atípica su presentación clínica? An Med Interna (Madrid) 2001; 18: 124-126.

\section{INTRODUCCIÓN}

La neumonía es una patología común en el paciente anciano, y se acompaña en estos casos de mayor morbi-mortalidad. Generalmente, según aumenta la edad, son mayores las dificultades diagnósticas de esta entidad (1). Este echo se ha puesto en relación con varios factores, entre los que destacan; disminución en la presentación de los síntomas típicos, formas atípicas de presentación, dificultad en la anamnesis, factores comórbidos de confusión y mayor prevalencia de altera- ciones crónicas en las radiografías de tórax. Estos factores conllevan con frecuencia un retraso en el diagnóstico, con empeoramiento añadido del pronóstico $(1,2)$.

Recientemente, varios estudios han puesto en duda el carácter frecuentemente atípico de la clínica en la neumonía del anciano (3-6). Otros refieren una disminución general de los síntomas en general (respiratorios y no respiratorios), aunque sin clara variación de la sintomatología con respecto a grupos de menor edad (7). Sin embargo, parece evidente la utilidad de conocer de forma adecuada las características clí-

Trabajo aceptado: 14 de Septiembre de 2000

Correspondencia: S. González Quijada. Avda. Cid Campeador 96. Hospital General Yagüe. Sección de Medicina Interna, 8 a Planta. 09005 Burgos. 
nicas de esta entidad en este grupo de edad, sabiendo que ello va a repercutir en un correcto diagnóstico y tratamiento.

Por los motivos señalados decidimos estudiar las características de presentación clínica de las neumonías en nuestros pacientes de mayor edad y su posible relación con los antecedentes clínicos del paciente.

\section{PACIENTES Y MÉTODO}

Se revisaron de manera retrospectiva todos los casos con diagnóstico de neumonía en pacientes mayores de 65 años e ingresados durante 1992 en nuestro hospital. Se incluyeron aquellos pacientes que presentaban un cuadro clínico compatible junto con la presencia de un infiltrado pulmonar en la radiografía de tórax no conocido previamente. Para ello, dos radiólogos sin conocer la historia clínica, revisaron todas las radiografías del paciente, las actuales y previas que figuraban en el sobre de historia clínica. Se descartaron aquellos casos que podrían haberse explicado por otras patologías o de diagnóstico impreciso. Un internista experimentado revisó la historia de urgencias, los evolutivos y la historia de la planta, para delimitar las formas clínicas predominantes de presentación. Se establecieron cinco patrones: respiratorio (tos, expectoración, disnea, dolor, hemoptisis), febril (fiebre, escalofríos, tiritona), mental-neurológico (confusión, somnolencia, desorientación, coma), alteración en la deambulación-deterioro del estado general (disminución de la actividad y deambulación, astenia, anorexia, apatía, deterioro del estado general) y abdominal digestivo (nauseas, vómitos, dolor abdominal, diarrea). Se consideró como patrón clínico típico el "febril-respiratorio", según que cumpliera los criterios comúnmente aceptados para el diagnóstico de neumonia (8). Se definió como cuadro clínico dominante aquel que cumplía los siguientes criterios: a) Era el motivo principal de consulta o de acudir a urgencias. b) Figuraba como cuadro principal en la historia clínica. c) Motivó la petición de interconsulta al especialista. d) No excluía la aparición de otros síntomas relacionados con otro órgano o sistema.

Otros internistas recopilaron el resto de datos a estudio; características del paciente, tiempo de evolución de los síntomas y antecedentes (siempre contrastado informes previos). Se incluyeron la presencia de fiebre, hipotensión (TA sistólica $<90 \mathrm{mmHg}$ ) e insuficiencia respiratoria (saturación de oxígeno en sangre arterial menor del $84 \%$ ) como variables de confusión, por la posibilidad de que su presencia se relacionara con clínica neurológica.

Los datos continuos han sido expresados mediante la media y la desviación estándar (SD), y los dicotómicos en frecuencias relativas o porcentajes. Los métodos estadísticos utilizados fueron $\mathrm{X}^{2}$ y prueba exacta de Ficher para la comparación de variables cualitativas, y t de Student para cuantitativas. Se emplearon modelos de análisis múltiple (regresión logística) para el estudio de las asociaciones entre los antecedentes, características y los patrones clínicos. En todos los casos se consideró el test realizado como estadísticamente significativo cuando p era menor de 0,05 , es decir, para un nivel de confianza del $95 \%$.

\section{RESULTADOS}

Se revisaron 203 historias de las que fueron incluidas en el estudio 179. Los pacientes presentaban una edad media de 78 años, siendo la proporción de varones del 65,9\%. El 91\% presentaban neumonías adquiridas en la comunidad. Se obtuvo diagnóstico microbiológico en el $20 \%$ de los casos empleando las técnicas habituales. La proporción de pacientes según las características, el tiempo de evolución y antecedentes comórbidos figuran en la Tabla I.

TABLA I

CARACTERÍSTICAS Y ANTECEDENTES DE LOS PACIENTES

\begin{tabular}{|c|c|c|c|}
\hline & $\begin{array}{l}\text { Total } \\
(n=179)\end{array}$ & $\begin{array}{l}65-75 \text { años } \\
(n=62)\end{array}$ & $\begin{array}{l}>75 \text { años } \\
(n=117)\end{array}$ \\
\hline \multicolumn{4}{|l|}{ Características } \\
\hline Edad (años) $)^{a}$ & $78,4(9,5)$ & $69,9(3,0)$ & $82,9(4,8)$ \\
\hline Sexo varón & 65 & 74 & 61 \\
\hline Procedencia de la comunidad & $91(163)$ & $87,1(54)$ & $93,1(109)$ \\
\hline Fiebre $\left(>37^{\circ} \mathrm{C}\right)$ & $70,9(127)$ & $73,0(45)$ & $70,0(82)$ \\
\hline Fiebre $\left(>38^{\circ} \mathrm{C}\right)$ & $48,0(86)$ & $52,0(32)$ & $46,0(54)$ \\
\hline Insuficiencia respiratoria & $24,0(43)$ & $24,0(15)$ & $24,0(28)$ \\
\hline Hipotensión & $6,7(12)$ & $3,0(2)$ & $9,0(10)$ \\
\hline Antibioterapia previa & $12,9(23)$ & $8,0(5)$ & $15,0(18)$ \\
\hline \multicolumn{4}{|l|}{ Tiempo de evolución de los síntomas } \\
\hline Clínica $<48$ h. evolución & $27,6(49)$ & $28,0(17)$ & $28(32)$ \\
\hline Clínica > 7 días evolución & $35,0(62)$ & $41,0(25)$ & $32,0(37)$ \\
\hline \multicolumn{4}{|l|}{ Situación comórbida } \\
\hline Enfermedad pulmonar & $33,1(59)$ & $40,9(25)$ & $29,0(34)$ \\
\hline Enfermedad cardiovascular & $30,1(54)$ & $24,1(15)$ & $33.3(39)$ \\
\hline Inmunosupresión & $14,0(25)$ & $14,7(9)$ & $13,6(16)$ \\
\hline Enfermedad mental/neurológica ${ }^{b}$ & $25,2(45)$ & $14,7(9)$ & $30,7(36)$ \\
\hline Deambulación alterada & $8,9(16)$ & $3,2(2)$ & $11,9(14)$ \\
\hline Sin antecedentes valorables & $15,0(27)$ & $17,7(11)$ & $13,6(16)$ \\
\hline
\end{tabular}

Excepto la edad, las demás variables se expresan en porcentajes y en paréntesis el número de pacientes. ${ }^{a} p<0,001$ entre los dos subgrupos; ${ }^{b} p<0,05$ entre los dos subgrupos. 
El 73,1\% de los pacientes mostraron una presentación clínica que se puede considerar típica (febril-respiratoria), mientras que el $26,8 \%$ presentaban patrones atípicos. La proporción de los distintos patrones de presentación clínica se muestran en la Tabla II. Se realizó un análisis por subgrupos de edad (65-75 y >75 años) que se muestran en las dos tablas. El porcentaje de los patrones de presentación clínica atípicos (mental-neurológico, deambulación-estado general, digestivoabdominal) fue superior en le subgrupo de pacientes mayores de 75 años, aunque sin mostrar diferencias significativas. La forma de presentación respiratoria se asoció de forma directa e independiente con la presencia de antecedentes respiratorios $(\mathrm{p}<0,001)$ y de forma inversa con antecedentes mentales-neurológicos $(\mathrm{p}<0,05)$. El patrón febril se asoció con la ausencia de antecedentes cardiovasculares $(p<0,05)$ y el mental-neurológico con la presencia de antecedentes neurológicos $(\mathrm{p}<0,00)$. La presentación como alteración de la deambulación-estado general se asoció con un tiempo de evolución mayor de 7 días $(\mathrm{p}<0,05)$.

TABLA II

PATRO NES PREDO M INANTES DE PRESENTACIÓ N CLÍNICA

\begin{tabular}{llll}
\hline & $\begin{array}{l}\text { Total } \\
(\mathrm{n}=179)\end{array}$ & $\begin{array}{l}65-75 \text { años } \\
(\mathrm{n}=62)\end{array}$ & $\begin{array}{l}>75 \text { años } \\
(\mathrm{n}=117)\end{array}$ \\
\hline Respiratorio & $47,4(85)$ & $54,8(34)$ & $43,5(51)$ \\
Febril & $25,7(179)$ & $29,0(18)$ & $23,9(28)$ \\
M ental/neurológico & $10,6(19)$ & $6,4(4)$ & $12,8(15)$ \\
Deambulación/estado general & $6,15(11)$ & $3,2(2)$ & $7,6(9)$ \\
Abdominal/digestivo & $9,5(17)$ & $4,8(3)$ & $11,9(14)$
\end{tabular}

Los resultados se expresan en porcentajes y en paréntesis el número de pacientes

\section{DISCUSIÓN}

El cuadro clínico de la neumonía en el anciano puede presentarse de forma habitual con fiebre, tos, expectoración y dolor pleurítico, o de forma atípica $(1,2)$. Entre los síntomas referidos en estas formas de presentación no habitual figuran la alteración de la condición general, falta de fiebre, deterioro de la función basal, confusión y descompensación de la enfermedad subyacente (1). Debido a esta falta de síntomas específicos con frecuencia la neumonía es infradiagnosticada, pudiendo asociarse una mayor morbimortalidad (9).

Desde Osler (10), varios estudios han reafirmado el carácter "atípico" en la presentación de esta patología en los ancianos. Sin embargo, un análisis detenido de varias series posteriores, contradicen esta postura tradicional. Así, Riquelme et al, encuentran que el $77 \%$ de sus pacientes de mayor edad presentaron una clínica habitual (11), y Llorente et al y Venkatesan et al describen una presentación típica en el 86 y el 55\% de los casos, respectivamente $(6,12)$. Incluso en un estudio comparativo entre pacientes con neumonía neumocócica bacteriémica, apenas hubo diferencias entre los datos clínicos de los ancianos y los menores de 65 años (3). Recientemente Metlay u cols., en un gran estudio prospectivo que abarcaba pacientes desde los 18 años, concluyen afirmando que tanto los síntomas respiratorios como los no respiratorios son menos comunes que los habitualmente descritos (7). Sin embargo, estos autores no contemplan el análisis de síntomas indicativos de disfunción cerebral, siendo estos los que tradicionalmente se han considerado característicos como formas de presentación en los ancianos (10). Quizás, la controversia que se ha generado, radica más en la interpretación que cada autor da al porcentaje que considera el adecuado de la presencia de los síntomas típicos/atípicos en este tipo de pacientes.

En nuestro estudio, en vez de un análisis de los síntomas aislados, que puede generar este tipo de discusión, describimos el porcentaje de patrones distintivos de presentación. Pensamos que esta orientación es más aproximada a la práctica clínica habitual. Así ya, Osler, denominó inicialmente esta patología en los ancianos como "neumonía senil", basándose en el patrón de baja temperatura y síntomas cerebrales (10). Según este enfoque, un cuarto de los pacientes mayores de 65 años, y un tercio de aquellos mayores de 75 años, cursan con patrones clínicos atípicos predominantes de presentación. Observamos además, que estos están en estrecha relación con los antecedentes patológicos, por aparato o sistema, del paciente. Parece que la clínica predominante esta en relación con la descompensación de la patología previa, como ya se había descrito en otras patologías infecciosas en esta edad $(13,14)$.

\section{Bibliografía}

1. Fein AM. Pneumonia in the elderly. Special diagnostics and therapeutic considerations. Med Clin North Am 1994; 78: 1015-33.

2. Zalacain R, Camino J, Cabriada V. Pneumonia in the elderly. Arch Bronconeumol 1998; 34: 63-67.

3. Esposito AL. Community-acquired bacteremic pneumococcal pneumonia: effect of age in manifestations and outcome. Arch Intern Med 1984; 144: 945-948.

4. Marrie TJ, Haldane EV, Faulkner RS, Durant H, Kwan C. Communityacquired pneumonia requiring hospitalization: is it different in the elderly? J Am Geriatr Soc 1985; 33: 671-680.

5. Harper C, Newton P. Clinical aspects of pneumonia in the elderly veteran. J Am Geriatr Soc 1989; 37: 867-872.

6. Venkatesan P, Gladman J, Macfarlane JT, Barer D, Berman P, Kinner $\mathrm{W}$ et al. A hospital study of community acquired pneumonia in the elderly. Thorax 1990; 45: 254-258.

7. Metlay JP, Schulz R, Li YH, Singer DE, Marrie IJ, Coley CM et al. Influence of age on symptoms at presentation in patients with community-acquired pneuminia. Arh Intern Med 1997; 157: 1453-1459.
8. Chow AW, Hall CB, Klein JO, Kammer RB, Meyer RD, Remington JS. Evaluation of new-infective drugs for the treatment of respiratory tract infections. Infections diseases Society of America and the Food and Drug Administration. Clin Infect Dis 1992; 15: 62-88.

9. Whitson B, Campbell GD. Community-acquired pneumonia: new outpatient guidelines on age, severty of illness. Geriatrics 1994; 49: 24-36.

10. Osler W, ed. The principles and Practice of Medicine. 3rd ed. New York: D. Appleton 1898; 109.

11. Riquelme R, Torres A, El-Ebiary M, Mensa J, Estruch R, Ruiz M et al. Community-acquired pneumonia in the elderly. Clínical and nutritional aspects. Am J Respir Crit Care Med 1997; 156: 1908-1914.

12. Llorente JL, Zalacain R, Gaztelurrutia, Talayero N, Pérz M, Badiol C et al. Características clínicas y etiológicas de la neumonía adquirida en la comunidad en ancianos. Enferm Infecc Microbiol Clin 1994; 12: 21-25.

13. Garibaldi RA, Nurse BA. Infection in the elderly. Am J Med 1986; 81: 53-58.

14. Fein AM, Feinsilver SH, Niederman MS. Atypical manifestations of pneumonia in the elderly. Clin Chest Med 1991; 12: 319-336. 